\title{
On topological type of periodic self-homeomorphisms of closed non-orientable surfaces
}

\author{
G. Gromadzki ${ }^{1}$ - B. Szepietowski ${ }^{1}$
}

Received: 31 July 2014 / Accepted: 28 June 2015 / Published online: 14 July 2015

(C) The Author(s) 2015. This article is published with open access at Springerlink.com

\begin{abstract}
Let $S_{g}$ denote a closed non-orientable surface of genus $g \geq 3$. At the beginning of 1980s E. Bujalance showed that the maximum order of a periodic self-homeomorphism of $S_{g}$ is equal to $2 g$ or $2(g-1)$ for $g$ odd or even respectively, and this upper bound is attained for all $g \geq 3$. In this paper we enumerate, up to topological conjugation, actions on $S_{g}$ of a cyclic group $\mathbb{Z}_{N}$ of order $N>g-2$ with prescribed type of the quotient orbifold $S_{g} / \mathbb{Z}_{N}$. We also compute, for a fixed $g$ and $N$ ranging between $\max \{g, 3(g-2) / 2\}$ and $2 g$, the total numbers of different topological types of action of $\mathbb{Z}_{N}$ on $S_{g}$.
\end{abstract}

Keywords Closed non-orientable surfaces - Topological classification of periodic self-homeomorphisms · Mapping class group $\cdot$ Klein surfaces

Mathematics Subject Classification Primary 30F; Secondary 57M · 14H

\section{Introduction}

By an effective action of a finite group $G$ on a closed surface $S$ we understand an embedding of $G$ into the group $\operatorname{Homeo}(S)$ of homeomorphisms of $S$. Two such actions are topologically equivalent, or of the same topological type, if the images of $G$ are conjugate in $\operatorname{Homeo}(S)$. The topological classification of finite group actions on closed surfaces is a classical problem going back to Nielsen [16].

G. Gromadzki and B. Szepietowski supported by NCN 2012/05/B/ST1/02171. G. Gromadzki supported also by Max-Planck Mathematical Institute in Bonn.

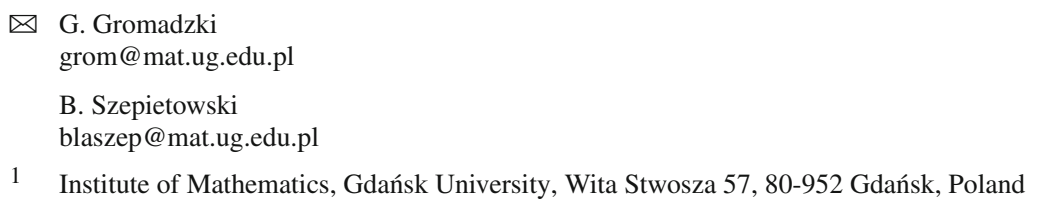


Let $\mathcal{M}_{g}$ denote the moduli space of complex algebraic curves of genus $g \geq 2$ and consider its subset $\mathcal{M}_{g}(G)$ consisting of points representing curves with a finite group $G$ of birational automorphisms. It is intuitively plausible, and Teichmüller-Royden theory provides a more precise justification, that $\mathcal{M}_{g}(G)$ is smaller for bigger $G$. In other words, a curve is better described by its group of automorphims when this group is bigger. By the famous Hurwitz bound its order does not exceed 84 $(g-1)$.

Particularly interesting are the cases when a curve $X$ is determined, up to birational equivalence, by the topological type of the action of $G$, or only by its ramification data, by which we understand the genus of the orbit space $X / G$ and the branching indices of the projection $X \rightarrow X / G$, or even only by the order of $G$. More specifically, when $|G|>$ $12(g-1)$, then by the Hurwitz-Riemann formula and elementary Teichmüller theory, $\mathcal{M}_{g}(G)$ is finite and topological and birational types of the action coincide, see $[9,12,17,18]$. For example, the main discovery of Nakagawa from [15], independently proved also by Hirose in [13] using less explicit methods which are closer to our appproach, asserts that with a few exceptions, a complex curve of genus $g \geq 2$ having an automorphism of order $N \geq 3 g$ is determined (up to birational equivalence) by $N$. The condition $N \geq 3 g$ turns out to be quite restrictive, as it forces $N$ to be one of $4 g+2,4 g, 3 g+3$ or $3 g$ for $g>12$. On the other hand, there are infinitely many rational numbers $q$ and $r$, such that for infinitely many $g \geq 2$ there is a homeomorphism of a closed orientable surface of genus $g$ having order $N=q g+r$ satisfying $2(g-1)<N<3 g$. In [1] a more general situation is studied, when the order of a cyclic group of automorphisms of a compact Riemann surface of genus $g \geq 2$, or the ramification data of the action, determine its topological type. Importance of such results follows from their connection with topology of the singular locus of the moduli space of complex algebraic curves, see [11].

Motivated by $[1,13]$, in this paper we consider analogous problems for purely imaginary real curves, which can also be seen as compact, unbordered, non-orientable surfaces with dianalitic structure (see [5] for a definition). The study of dianalitic automorphisms of such surfaces is equivalent to the study of their periodic self-homeomorphisms, because every periodic homeomorphism of a surface $S_{g}$ of topological genus $g \geq 3$ is a dianalitic automorphism with respect to some dianalitic structure on $S_{g}$. Bujalance showed in [2] (see also later paper of S. Wang [19]) that the maximal order of such automorphism of a non-orientable surface of genus $g \geq 3$ is equal to $2 g$ or $2(g-1)$ for $g$ odd or even respectively, and this upper bound is attained for all $g \geq 3$. The case $g=3$ is well understood, as the mapping class group of $S_{3}$ is isomorphic to $\mathrm{GL}_{2}(\mathbb{Z})$ (see [8]), and the classification of conjugacy classes of torsion elements in the latter group is known. Another interesting problem concerning cyclic periodic actions on non-orientable surfaces was considered in recent paper [3], where the authors investigated such actions which can not be extended to any bigger group.

Throughout the whole paper we denote by $S$ or $S_{g}$ a closed (i.e. compact and without boundary) non-orientable surface of genus $g \geq 3$. In this paper we study the extent to which the order $N$ or the ramification data of a cyclic group $G$ acting on $S_{g}$ determine the topological type of the action, which is important in virtue of the connection with topological properties of moduli spaces of purely imaginary real algebraic curves, similar as in the case of orientable surfaces. More specifically, in Sect. 3 we investigate rigidity of topological type of cyclic group actions of order $N>g-2$ with prescribed ramification data. We consider a quite large family of actions, where the order $N$ has the form $N=q g+r$, for infinitely many rational $q$ and $r$. Furthermore, for each such pair $q, r$, there is an action of $\mathbb{Z}_{N}$ on $S_{g}$ for infinitely many genera $g$. As an application, in Sect. 4 we calculate, for a fixed $g$ and all $N$ between $\max \left\{g, \frac{3}{2}(g-2)\right\}$ and $2 g$, the numbers of topological types of action on $S_{g}$ of a 
cyclic group of order $N$. This should be seen as an analogue, for a non-orientable surface, of the main result of [13].

\section{Preliminaries}

\subsection{Principal definitions}

Our approach is based on algebraic properties of discrete subgroups of isometries of the hyperbolic plane $\mathcal{H}$, called NEC-groups. We refer the reader to the monograph [5] for an extensive exposition of the theory.

Suppose that a finite group $G$ acts effectively by homeomorphisms on a closed nonorientable surface $S=S_{g}$ of genus $g \geq 3$. Fix a dinanalitic structure on $S$, with respect to which $G$ acts by dianalitic automorphisms. Then $S$ is conformally isomorphic to the orbit space $\mathcal{H} / \Gamma$ for a torsion-free NEC group $\Gamma$ isomorphic to $\pi_{1}(S)$. Such $\Gamma$ is called nonorientable surface group. Furthermore, $G$ is isomorphic to the quotient $\Lambda / \Gamma$, for some other NEC-group $\Lambda$, a subgroup of the normalizer of $\Gamma$ in the group of all isometries of $\mathcal{H}(\Lambda$ is equal to that normaliser if and only if $G$ is the full group of dianalitic automorphisms of $S$ ). Equivalently, there is an epimorphism $\theta: \Lambda \rightarrow G$ with kernel $\Gamma$, usually called smooth epimorphism to underline the fact that its kernel is torsion-free. This motivates the following definition.

Definition 2.1 Suppose that $\Lambda$ is an NEC group, $G$ is a finite group, and $\theta: \Lambda \rightarrow G$ is an epimorphism. We say that $\theta$ is an NSK-map (non-orientable-surface-kernel-map) if and only if $\operatorname{ker} \theta$ is a non-orientable surface group.

Two effective actions of $G$ on $S_{g}$ are topologically conjugate (by a homeomorphism of $S_{g}$ ) if and only if the associated NSK-maps are equivalent in the sense of the next definition (see [4, Proposition 2.2] and its proof; the same argument applies to closed surfaces).

Definition 2.2 We say that two NSK-maps $\theta_{i}: \Lambda_{i} \rightarrow G, i=1,2$, are equivalent if and only if there exist isomorphisms $\phi: \Lambda_{1} \rightarrow \Lambda_{2}$ and $\alpha: G \rightarrow G$ such that the following diagram is commutative.

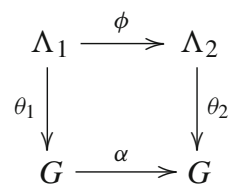

The ramification data of $G$ is encoded in the signature $\sigma(\Lambda)$ of $\Lambda$, which in our case has the form

$$
\left(h ; \pm ;\left[m_{1}, \ldots, m_{r}\right] ;\{(), . k,()\}\right),
$$

where $k>0$ if the sign is "+" (see [2]). The orbit space $S / G=\mathcal{H} / \Lambda$ has genus $h$ and $k$ boundary components, and it is orientable if and only if the sign is " + ". From the signature one can also read a presentation of $\Lambda$ in terms of canonical generators and defining relations as follows. The generators are:

$$
\begin{array}{ll}
x_{i} & \text { for } 1 \leq i \leq r \\
c_{j}, e_{j} & \text { for } 1 \leq j \leq k \\
a_{l}, b_{l} & \text { for } 1 \leq l \leq \mathrm{h} \text { if the sign is " }+" \\
d_{l} & \text { for } 1 \leq l \leq \mathrm{h} \text { if the sign is " }-"
\end{array}
$$


The defining relations are:

$$
\begin{array}{ll}
x_{i}^{m_{i}}=1 & \text { for } 1 \leq i \leq r \\
c_{j}^{2}=1,\left[e_{j}, c_{j}\right]=1 & \text { for } 1 \leq j \leq k \\
x_{1} \cdots x_{r} e_{1} \cdots e_{k}\left[a_{1}, b_{1}\right] \cdots\left[a_{h}, b_{h}\right]=1 & \text { if the sign is " }+" \\
x_{1} \cdots x_{r} e_{1} \cdots e_{k} d_{1}^{2} \cdots d_{h}^{2}=1 & \text { if the sign is " }-"
\end{array}
$$

Note that $\Lambda$ is a proper NEC group, i.e. it contains orientation-reversing isometries. Among the canonical generators, $c_{j}$ and $d_{l}$ are orientation-reversing, the remaining ones are orientation-preserving. We denote by $\Lambda^{+}$the canonical Fuchsian subgroup of $\Lambda$, consisting of all orientation-preserving elements of $\Lambda$. Finally, we have the Hurwitz-Riemann ramification formula

$$
g-2=|G| \mu(\sigma),
$$

where

$$
\mu(\sigma)=\alpha h-2+k+\sum_{i=1}^{r}\left(1-\frac{1}{m_{i}}\right)
$$

is the normalized hyperbolic area of a (arbitrary) fundamental region for $\Lambda$. Here $\alpha=2$ if the sign of the signature is "+" and $\alpha=1$ otherwise.

The following lemma, which is a special case of [2, Proposition 3.2], provides an effective criterion for an NSK-map.

Lemma 2.3 Suppose that $\Lambda$ is an NEC group with signature (2.2). A group homomorphism $\theta: \Lambda \rightarrow G$ is an NSK-map if and only if

1. $\theta\left(x_{i}\right)$ has order $m_{i}$ for $1 \leq i \leq r$,

2. $\theta\left(c_{j}\right)$ has order 2 for $1 \leq i \leq k$,

3. $\theta\left(\Lambda^{+}\right)=G$.

In this paper we are interested in the case where $G$ is a cyclic group $\mathbb{Z}_{N}$.

Definition 2.4 Suppose that $\sigma$ is an NEC signature (2.2) and $N$ is a positive integer. We say that the pair $(\sigma, N)$ is admissible if there exists a NSK-map $\theta: \Lambda \rightarrow \mathbb{Z}_{N}$ with $\sigma(\Lambda)=\sigma$. If, furthermore, such $\theta$ is unique up to equivalence, then we say that $(\sigma, N)$ is rigid.

\subsection{Automorphisms of NEC-groups vs mapping class groups}

In this subsection we recall the relationship between the outer automorphism group of an NEC-group $\Lambda$ and the mapping class group of the orbit space $\mathcal{H} / \Lambda$. The outer automorphism $\operatorname{group} \operatorname{Out}(\Lambda)$ is the quotient $\operatorname{Aut}(\Lambda) / \operatorname{Inn}(\Lambda)$ of the group of all automorphisms of $\Lambda$ by the subgroup of inner automorphisms. For simplicity we assume that the signature of $\Lambda$ has the form

$$
\left(h ;-;\left[m_{1}, \ldots, m_{r}\right] ;\{-\}\right),
$$

where the periods $m_{i}$ are all different. For a discussion of the general case see [4, Section 4].

Set $S=\mathcal{H} / \Lambda$ and note that $S$ is a non-orientable surface of genus $h$ with $r$ distinguished points, over which the projection $p: \mathcal{H} \rightarrow S$ is ramified. Let $\mathcal{P}$ denote the set of distinguished points, $\mathcal{U}=\mathcal{H} \backslash p^{-1}(\mathcal{P})$ and $S_{0}=S \backslash \mathcal{P}$. Then $p: \mathcal{U} \rightarrow S_{0}$ is a regular covering and $\Lambda$ is its deck group isomorphic to $\pi_{1}\left(S_{0}\right) / p_{*}\left(\pi_{1}(\mathcal{U})\right)$. The canonical generators $x_{1}, \ldots, x_{r}$ and 
$d_{1}, \ldots, d_{h}$ of $\Lambda$ correspond to standard generators of $\pi_{1}\left(S_{0}\right)$ and $p_{*}\left(\pi_{1}(\mathcal{U})\right)$ is normally generated by $x_{i}^{m_{i}}$ for $i=1, \ldots, r$.

We denote by $\operatorname{Mod}(S, \mathcal{P})$ the mapping class group of $S$ relative to $\mathcal{P}$, defined as the group of isotopy classes of homomorphism of $S$ preserving $\mathcal{P}$. The pure mapping class group is the subgroup $\operatorname{PMod}(S, \mathcal{P})$ of $\operatorname{Mod}(S, \mathcal{P})$ consisting of the isotopy classes of homomorphism fixing each element of $\mathcal{P}$. The groups $\operatorname{PMod}(S, \mathcal{P})$ and $\operatorname{Out}(\Lambda)$ are isomorphic by a generalisation, for non-orientable $S$, of [12, Theorem 1] (see [7, Section 3]). Given an element of $\operatorname{PMod}(S, \mathcal{P})$ one can find its image in $\operatorname{Out}(\Lambda)$ as follows. Represent this element by a homeomorphism $f: S_{0} \rightarrow S_{0}$ fixing some base point. Then $f_{*}: \pi_{1}\left(S_{0}\right) \rightarrow \pi_{1}\left(S_{0}\right)$ preserves $p_{*}\left(\pi_{1}(\mathcal{U})\right)$, hence it induces an automorphism of the quotient $\pi_{1}\left(S_{0}\right) / p_{*}\left(\pi_{1}(\mathcal{U})\right) \cong \Lambda$.

\subsection{Some elementary algebra}

In this paper we use additive notation for cyclic groups. For two integers $a, b$ we denote by $(a, b)$ their greatest common divisor, $\varphi$ denotes the Euler's totient function. We will use the following version of Chinese Remainder Theorem.

Lemma 2.5 Given integers $a, b$, the system of congruences

$$
\left\{\begin{array}{l}
x \equiv a(m) \\
x \equiv b(n)
\end{array}\right.
$$

has a solution if and only if $a \equiv b(d)$, where $d=(m, n)$ and this solution is unique up to $\operatorname{lcm}(m, n)$.

By abuse of language we will write $a \in \mathbb{Z}_{N}=\mathbb{Z} / N \mathbb{Z}$ for a non-negative integer $a<N$. Given positive integers $x$ and $m$ we denote by $[x]_{m}$ the reduction of $x$ modulo $m$.

\section{Topological type of actions of big order}

Suppose that $\mathbb{Z}_{N}$ acts on a closed non-orientable surface $S_{g}$ of genus $g \geq 3$. Then there is an NEC group $\Lambda$ and an NSK-map $\theta: \Lambda \rightarrow \mathbb{Z}_{N}$ such that $g-2=N \mu(\sigma)$, where $\sigma$ is the signature of $\Lambda$. In particular, $N>g-2$ if and only if $\mu(\sigma)<1$. The next lemma follows by inspection.

Lemma 3.1 If $N>g-2$ then $\Lambda$ has one of the following signatures:

$$
\begin{gathered}
(2 ;-;[m] ;\{-\}),(0 ;+;[m] ;\{(),()\}),(1 ;-;[m] ;\{()\}), \\
\left(0 ;+;\left[m_{1}, m_{2}\right] ;\{()\}\right),\left(1 ;-;\left[m_{1}, m_{2}\right] ;\{-\}\right), \\
(0 ;+;[2, p, q] ;\{()\}),(1 ;-;[2, p, q] ;\{-\}),
\end{gathered}
$$

where $p=2$ and $q$ arbitrary or $p=3$ and $q=3,4,5$.

Corollary 3.2 Suppose that $\mathbb{Z}_{N}$ acts on a closed non-orientable surface $S_{g}$ of genus $g \geq 3$. If $N>g-2$ then $S_{g} / \mathbb{Z}_{N}$ is one of the following orbifolds:

- Klein bottle with 1 cone point,

- annulus with 1 cone point,

- Möbius strip with 1 cone point,

- disc with 2 or 3 cone points,

- projective plane with 2 or 3 cone points. 
In this section we determine some rigid pairs $(\sigma, N)$ for the signatures listed in Lemma 3.1 and compute the numbers of equivalence classes of NSK-maps. The results presented here are of three types. The first type concerns necessary and sufficient conditions for $(\sigma, N)$ to be admissible - most of them follow from a more general result in [10], where such conditions are given for an arbitrary NEC signature $\sigma$. The second type of results concerns automorphisms of NEC-groups, which are related to mappings class groups of surfaces from Corollary 3.2. Some of these results are borrowed from [4] and some are new. Finally we state, in the form of corollaries, topological consequences of our results, which will play a key role in Sect. 4 , and we believe that they are of independent interest.

\subsection{Actions with a 1-punctured Klein bottle as the quotient orbifold}

In this subsection we fix $\Lambda$ with signature $(2 ;-;[m] ;\{-\})$. We also fix canonical generators $d_{1}, d_{2}$ of $\Lambda$, satisfying single defining relation $\left(d_{1}^{2} d_{2}^{2}\right)^{m}=1$.

Lemma 3.3 Let $y=d_{1}, z=d_{1} d_{2}$. Out $(\Lambda)$ is generated by classes of automorphisms $\alpha, \beta$, $\gamma$ defined by

$$
\alpha:\left\{\begin{array}{l}
y \mapsto y z \\
z \mapsto z
\end{array} \quad \beta:\left\{\begin{array}{l}
y \mapsto y^{-1} \\
z \mapsto z
\end{array} \quad \gamma:\left\{\begin{array}{l}
y \mapsto y \\
z \mapsto z^{-1}
\end{array}\right.\right.\right.
$$

Proof Recall from Sect. 2.2 that $\operatorname{Out}(\Lambda)$ is isomorphic to the mapping class group $\operatorname{Mod}(S,\{P\})$, where $S=\mathcal{H} / \Lambda$ is a Klein bottle with one distinguished point $P \in S$. This surface is represented on Fig. 1 as a sphere with two crosscaps, which means that the interiors of the shaded discs should be removed, and then antipodals points in each of the resulting boundary components should be identified. We have an isomorphism $\Lambda \cong \pi_{1}(S \backslash\{P\}) /\left\langle\left\langle x^{m}\right\rangle\right\rangle$, where $\left\langle\left\langle x^{m}\right\rangle\right\rangle$ denotes the normal closure of $x^{m}=\left(d_{1}^{2} d_{2}^{2}\right)^{m}$ and $d_{1}, d_{2}$ are the standard generators of $\pi_{1}(S \backslash\{P\})$ shown on Fig. 1 (right). Let us briefly describe the generators of $\operatorname{Mod}(S,\{P\})$ given in [14]. Consider the simple closed curve $a$ on $S$ shown on Fig. 1 (left). Observe that $z=d_{1} d_{2}$ is represented by a simple loop freely homotopic to $a$. This curve is two-sided, which means that its regular neighbourhood is an annulus. Cutting $S$ along $a$, twisting one of the sides by $360^{\circ}$ in the direction indicated by the small arrows on the figure and gluing back gives a self-homeomorphism of $S$, whose isotopy class is denoted by $T_{a}$ and called a Dehn twist along $a$ (see [6] for a precise definition). For $i=1,2$ we denote by $V_{i}$ the isotopy class of a self-homeomorphism of $S$ obtained by sliding the puncture $P$ once along the loop $v_{i}$ shown on Fig. 1 (middle). These mapping classes are called puncture slides. Finally, we denote by $Y$ the crosscap slide defined to be the isotopy class of a self-homeomorphism of $S$ obtained by sliding the left crosscap once along $a$ (see [14] for
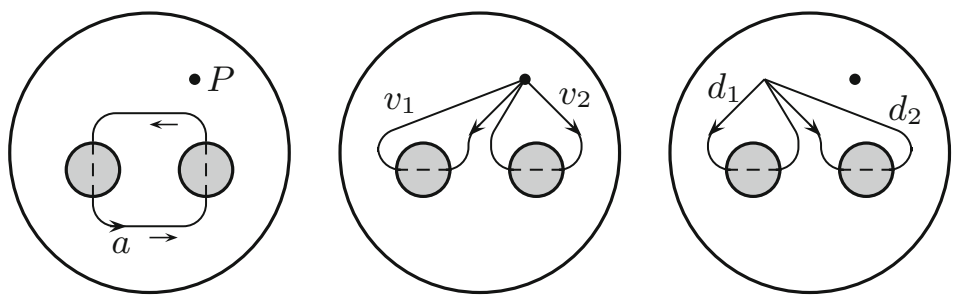

Fig. 1 Klein bottle with a puncture, the curve $a$ (left), the loops of the puncture slides (middle) and the generators of $\pi_{1}(S \backslash\{P\})$ (right) 
a precise definition). By [14, Theorem 4.9] $\operatorname{Mod}(S,\{P\})$ is generated by $\left\{T_{a}, V_{1}, V_{2}, Y\right\}$. Since $T_{a}\left(v_{1}\right)=v_{2}^{-1}$ up to isotopy fixing $P$, we have $V_{1}=T_{a}^{-1} V_{2}^{-1} T_{a}$ and the generator $V_{1}$ is redundant. By computing the automorphisms of $\pi_{1}(S \backslash\{P\})$ induced by $T_{a}, Y$ and $V_{2}$ we find that they represent the same elements of $\operatorname{Out}(\Lambda)$ as respectively $\alpha, \beta$ and $\gamma$.

The following lemma is a particular case of Lemma 5.9 in [10] for $r=1$.

Lemma $3.4(\sigma, N)$ is admissible if and only if either $m$ divides $N$ and $N$ is odd, or $2 m$ divides $N$ and $\frac{N}{2 m}$ is odd.

Proposition 3.5 Suppose that $(\sigma, N)$ is admissible, and let $d=\left(k, \frac{N}{k}\right)$, where $k=m$ if $N$ is odd or $k=2 m$ if $N$ is even. Then there are exactly $\left\lceil\frac{\varphi(d)}{2}\right\rceil$ equivalence classes of NSK-maps $\theta: \Lambda \rightarrow \mathbb{Z}_{N}$.

Proof Let $y$ and $z$ be the generators of $\Lambda$ from Lemma 3.3 and suppose that $\theta: \Lambda \rightarrow \mathbb{Z}_{N}$ is an NSK-map. Let $k$ denote the order of $\theta(z)$. We will see that $k=m$ for odd $N$, and $k=2 m$ for even $N$. By post-composing $\theta$ with an automorphism of $\mathbb{Z}_{N}$ we may assume $\theta(z)=N / k$. Set $a=\theta(y)$ and note that $a$ and $N / k$ must be coprime, because $\theta$ is an epimorphism. Furthermore, by pre-composing $\theta$ with a power of the automorphism $\alpha$ from Lemma 3.3, we may assume $a<N / k$, that is $a \in \mathbb{Z}_{N / k}^{*}$. Since $2 \theta(z)=\theta\left(d_{1}^{2} d_{2}^{2}\right)$ has order $m, k=m$ or $k=2 m$. Indeed, if $N$ is odd then $k=m$. If $N$ is even then $k=2 m$ for even $m$, whereas for odd $m$ either $k=m$ or $k=2 m$. Since $\Lambda^{+}$is generated by $z, y z y^{-1}$ and $y^{2}$, $\theta\left(\Lambda^{+}\right)$is generated by $N / k$ and $2 a$. By (3) of Lemma 2.3 these elements generate $\mathbb{Z}_{N}$, and thus $N / k$ is odd if $N$ is even, hence $k=2 m$.

Conversely, for any $a \in \mathbb{Z}_{N / k}^{*}$, the mapping $\theta_{a}: \Lambda \rightarrow \mathbb{Z}_{N}$ defined as $\theta_{a}(z)=N / k$ and $\theta_{a}(y)=a$ is an NSK-map. To finish the proof it suffices to show that for $a, b \in \mathbb{Z}_{N / k}^{*}, \theta_{a}$ and $\theta_{b}$ are equivalent if and only if $a \equiv \pm b(d)$.

Suppose that $a \equiv \varepsilon b(d)$, where $\varepsilon \in\{1,-1\}$ and $a, b \in \mathbb{Z}_{N / k}^{*}$. Let $b^{\prime}$ be the inverse of $b$ modulo $N / k$. Then $\varepsilon b^{\prime} a \equiv 1(d)$, and by Lemma 2.5 , there exists $c \in \mathbb{Z}_{N}$ such that $c \equiv 1(k)$ and $c \equiv \varepsilon b^{\prime} a(N / k)$. Observe that $c \in \mathbb{Z}_{N}^{*}$ and we have $c \theta_{b}(z)=N / k$ and $c \theta_{b}(y)=\varepsilon a+l N / k$ for some $l$. Then $c \theta_{b}=\theta_{a} \circ \alpha^{\varepsilon l} \circ \beta^{i}$, where $i=0$ or $i=1$ if $\varepsilon=1$ or $\varepsilon=-1$ respectively.

Suppose conversely, that $\theta_{a}$ and $\theta_{b}$ are equivalent, that is $c \theta_{b}=\theta_{a} \circ \phi$ for some $c \in \mathbb{Z}_{N}^{*}$ and $\phi \in \operatorname{Aut}(\Lambda)$. By Lemma 3.3 we have $\theta_{a} \phi(y)= \pm a+l \frac{N}{k}$ for some $l \in \mathbb{Z}_{k}$ and $\theta_{a} \phi(z)= \pm \frac{N}{k}$. From the former equality we have $c b= \pm a(N / k)$ and from the latter one $c= \pm 1(k)$ and it follows that $b= \pm a(d)$.

Corollary 3.6 There exists an action of $G=\mathbb{Z}_{N}$ on $S$ with $S / G$ being a Klein bottle with a single cone point of order $m$ if and only if $m$ and $N$ satisfy the conditions from Lemma 3.4 , and then the number of topological types of such action is $\left\lceil\frac{\varphi(d)}{2}\right\rceil$, where $d$ is as in Proposition 3.5. In particular, such action is unique up to topological conjugation if and only if $d \in\{1,3\}$.

\subsection{Actions with 1-punctured annulus or Möbius band as the quotient orbifold}

Here we fix NEC groups $\Lambda_{1}$ and $\Lambda_{2}$ with signatures $(0 ;+;[m] ;\{(),()\})$ and $(1 ;-;[m] ;\{()\})$, denoted respectively by $\sigma_{1}$ and $\sigma_{2}$. We also fix canonical generators $x, e, c_{1}, c_{2}$ of $\Lambda_{1}$, satisfying the following defining relations:

$$
x^{m}=c_{1}^{2}=c_{2}^{2}=1, \quad e c_{1}=c_{1} e, \quad x e c_{2}=c_{2} x e,
$$


and canonical generators $x, d, c$, of $\Lambda_{2}$, satisfying the following defining relations:

$$
x^{m}=c^{2}=1, \quad d^{2} x c=c d^{2} x .
$$

The following two lemmas are proved in [4, Lemma 4.6, Proposition 4.10 and Proposition 4.12].

Lemma 3.7 Out $\left(\Lambda_{1}\right)$ is isomorphic to the Klein four-group and is generated by classes of automorphisms $\alpha, \beta$ defined by

$$
\alpha:\left\{\begin{array}{l}
x \mapsto e^{-1} x^{-1} e \\
e \mapsto e^{-1} \\
c_{1} \mapsto c_{1} \\
c_{2} \mapsto c_{2}
\end{array} \quad \beta:\left\{\begin{array}{l}
x \mapsto e^{-1} x e \\
e \mapsto(x e)^{-1} \\
c_{1} \mapsto c_{2} \\
c_{2} \mapsto c_{1}
\end{array}\right.\right.
$$

Lemma 3.8 Out $\left(\Lambda_{2}\right)$ is isomorphic to the Klein four-group and is generated by classes of automorphisms $\gamma, \delta$ defined by

$$
\gamma:\left\{\begin{array}{l}
x \mapsto x^{-1} \\
d \mapsto x^{-1} d^{-1} x \\
c \mapsto c
\end{array} \quad \delta:\left\{\begin{array}{l}
x \mapsto x \\
d \mapsto(d x)^{-1} \\
c \mapsto(d x)^{-1} c(d x)
\end{array}\right.\right.
$$

Lemma 3.9 For $i=1,2,\left(\sigma_{i}, N\right)$ is admissible if and only if $N$ is even and $m$ divides $N$.

Proof The "only if" part follows immediately for Lemma 2.3. For the "if" part, assume that 2 divides $N, m$ divides $N$ and define $\theta_{i}: \Lambda_{i} \rightarrow \mathbb{Z}_{N}$ for $i=1,2$ by

$$
\begin{aligned}
& \theta_{1}(x)=\frac{N}{m}, \quad \theta_{1}(e)=1, \quad \theta\left(c_{j}\right)=\frac{N}{2} \text { for } j=1,2 \\
& \theta_{2}(x)=\frac{N}{m}, \quad \theta_{2}(d)=1+\frac{N}{2}, \quad \theta(c)=\frac{N}{2}
\end{aligned}
$$

Note that $\Lambda_{i}^{+}$is generated by conjugates of $x, e$ and $c_{1} c_{2}$ if $i=1$, and by conjugates of $x$ and $c d$ if $i=2$. It follows from Lemma 2.3 that $\theta_{i}$ are NSK-maps.

Remark 3.10 Similarly as a few other signatures consider in this section, the above signature $\sigma_{2}$ is a special case of the one from Lemma 5.14 in [10] for $r=1$. Unfortunately however, there is an error in the statement of that lemma, and we take the opportunity to correct it here: namely, the condition "and some of $N / 2, m_{1}, \ldots, m_{r}$ is even" must be deleted. In the proof, the authors failed to observe that $c_{0} d \in \Lambda^{+}$at the very end of page 182. Consequently, assertion (iv) of Theorem 6.4 in [10] also has to be modified. Its final part should read "where $\alpha=0$ if $\operatorname{lcm}\left(N / N_{1}, \ldots, N / N_{r}\right)=N$, and $\alpha=1$ otherwise."

The next lemma will be crucial for the proof of Proposition 3.12.

Lemma 3.11 Suppose that $m$ divides $N$ and $d=(m, N / m)$. Let $X$ be the subset of $\mathbb{Z}_{N}$ consisting of elements relatively prime to $\mathrm{N} / \mathrm{m}$, and let

$$
H=\left\{c \in \mathbb{Z}_{N}^{*}: c \equiv 1(m)\right\} .
$$

Then

(a) $H$ acts freely on $X$;

(b) the number of orbits is $\frac{m \varphi(d)}{d}$;

(c) $a, b \in X$ belong to the same orbit if and only if $a \equiv b(m)$. 
Proof (a) Note that $a \in X$ if and only if $a$ and $N / m$ generate $\mathbb{Z}_{N}$. For $a \in X$ and $c \in H$ we have $c a \in X$, because $c \frac{N}{m}=\frac{N}{m}$. To see that the action is free, suppose that $c a=a$ for $a \in X$ and $c \in H$. Write $c=1+\mathrm{km}$. We have that $N$ divides $a(c-1)$, hence $\frac{N}{m}$ divides $a k$. Since $\frac{N}{m}$ is relatively prime to $a$, it must divide $k$, hence $c=1$.

(b) Every element of $X$ is of the form $b+k \frac{N}{m}$ for $b \in \mathbb{Z}_{\frac{N}{m}}^{*}$ and $k \in \mathbb{Z}_{m}$. It follows that $X$ has $m \varphi(N / m)$ elements. The group $H$ is the kernel of the reduction epimorphism $\mathbb{Z}_{N}^{*} \rightarrow \mathbb{Z}_{m}^{*}$, hence $|H|=\varphi(N) / \varphi(m)$. Since the action of $H$ on $X$ is free, the number of orbits is $\frac{m \varphi(N / m) \varphi(m)}{\varphi(N)}=\frac{m \varphi(d)}{d}$.

(c) If $a, b \in X$ are in the same orbit, then clearly $a \equiv b(m)$. To prove the converse, it suffices to show that the set

$$
Y=\left\{b \in \mathbb{Z}_{m}: \exists_{a \in X} b=[a]_{m}\right\}
$$

has at least $\frac{m \varphi(d)}{d}$ elements. Fix $z \in \mathbb{Z}_{d}^{*}$, and choose $c \in \mathbb{Z}_{N / m}^{*}$ such that $z=[c]_{d}$, and any $b \in \mathbb{Z}_{m}$ such that $z=[b]_{d}$. By Lemma 2.5, there is $a \in X$ such that $a \equiv c(N / m)$ and $a \equiv b(m)$. Since there are $\varphi(d)$ choices for $z$ and $m / d$ choices for $b$, the assertion follows.

Proposition 3.12 Let $i \in\{1,2\}$ and suppose that $\left(\sigma_{i}, N\right)$ is admissible and $d=(\mathrm{m}, \mathrm{N} / \mathrm{m})$. The number of equivalence classes of NSK-maps $\Lambda_{i} \rightarrow \mathbb{Z}_{N}$ is

$$
\left\lceil\begin{array}{l}
\left.\frac{m \varphi(d)}{2 d}\right\rceil \text { if } d \neq 2 \text { or } 4 \nmid m, \\
\frac{m}{4}+1
\end{array} \text { if } d=2 \text { and } 4 \mid m .\right.
$$

Proof Let $X$ and $H$ be as in Lemma 3.11. For $a \in X$ we denote by $[a]$ the orbit of $a$ with respect to the action of $H$.

Case $i=1$. Every NSK-map $\Lambda_{1} \rightarrow \mathbb{Z}_{N}$ is equivalent, by multiplication by an element of $\mathbb{Z}_{N}^{*}$, to $\theta_{a}: \Lambda_{1} \rightarrow \mathbb{Z}_{N}$ defined by $\theta_{a}(x)=\frac{N}{m}, \theta_{a}\left(c_{j}\right)=\frac{N}{2}$ for $j=1,2$, and $\theta_{a}(e)=a$ for some $a \in X$.

For $a, b \in X, \theta_{a}$ is equivalent to $\theta_{b}$ if and only if $c \theta_{b}=\theta_{a} \circ \phi$ for some $c \in \mathbb{Z}_{N}^{*}$ and $\phi \in \operatorname{Aut}\left(\Lambda_{1}\right)$. By Lemma 3.7 we may assume $\phi \in\{1, \alpha, \beta, \alpha \beta\}$. Then, after replacing $c$ by $-c$ if necessary, we have $c \frac{N}{m}=\frac{N}{m}$ and either $c b=a$ or $c b=-\left(a+\frac{N}{m}\right)$. It follows that $\theta_{a}$ and $\theta_{b}$ are equivalent if and only if either $[b]=[a]$ or $[b]=\left[-a-\frac{N}{m}\right]$.

For $a \in X$ we say that the orbits $[a]$ and $\left[-a-\frac{N}{m}\right]$ are conjugate. If $[a]=\left[-a-\frac{N}{m}\right]$ then we say that $[a]$ is self-conjugate. Let $S$ denote the number of self-conjugate orbits, $T$ the total number of orbits given in (b) of Lemma 3.11, and $E$ the number of equivalence classes of NSK-maps. It follows from the previous paragraph that $E=(T+S) / 2$. Our next task is to compute $S$. By (c) of Lemma 3.11, $S$ is equal to the number of different $x \in \mathbb{Z}_{m}$ satisfying

$$
2 x \equiv-\frac{N}{m}(m)
$$

and such that $x=[a]_{m}$ for some $a \in X$. Evidently $S \leq 2$. We consider three cases.

Case $d>2$. Then $S=0$. For suppose that $x$ satisfies (3.1) and $x=[a]_{m}$ for some $a \in X$. Then $2 x \equiv 0(d)$ and since $a \in X, x$ is relatively prime to $d$, hence $d$ divides 2 which is a contradiction. In this case we have $E=T / 2=\frac{m \varphi(d)}{2 d}$.

Case $d=1$. Then $E=(m+S) / 2$. If $m$ is even then $\frac{N}{m}$ must be odd and (3.1) has no solution, hence $S=0$. If $m$ is odd then (3.1) has unique solution and $S=1$. 
Case $d=2$. Then (3.1) has two solutions, namely $x=\left[-\frac{N}{2 m}\right]_{m}$ and $x=\left[-\frac{N}{2 m}+\frac{m}{2}\right]_{m}$. Note, however, that $x=[a]_{m}$ for $a \in X$ if and only if $x$ is odd. If 4 divides $m$ then both solutions are odd, otherwise only one of them is odd. Thus, if $4 \nmid m$ then $S=1$ and $E=\frac{m+2}{4}=\left\lceil\frac{m}{4}\right\rceil$, while if 4 divides $m$ then $S=2$ and $E=\frac{m}{4}+1$.

Case $i=2$. Set $y=c d$. Every NSK-map $\Lambda_{2} \rightarrow \mathbb{Z}_{N}$ is equivalent, by multiplication by an element of $\mathbb{Z}_{N}^{*}$, to $\theta_{a}: \Lambda_{2} \rightarrow \mathbb{Z}_{N}$ defined by $\theta_{a}(x)=\frac{N}{m}, \theta_{a}(c)=\frac{N}{2}$ and $\theta_{a}(d)=a$ for some $a \in X$ (note that $\theta_{a}\left(\Lambda_{2}^{+}\right)$is generated by $\frac{N}{m}$ and $a$, hence the condition $a \in X$ is equivalent to (3) of Lemma 2.3).

For $a, b \in X, \theta_{a}$ is equivalent to $\theta_{b}$ if and only if $c \theta_{b}=\theta_{a} \circ \phi$ for some $c \in \mathbb{Z}_{N}^{*}$ and $\phi \in \operatorname{Aut}\left(\Lambda_{2}\right)$. By Lemma 3.7 we may assume $\phi \in\{1, \gamma, \delta, \gamma \delta\}$. Then, after replacing $c$ by $-c$ if necessary, we have $c \frac{N}{m}=\frac{N}{m}$ and either $c b=a$ or $c b=-\left(a+\frac{N}{m}\right)$. As in the case $i=1$, the number of equivalence classes of NSK-maps $\Lambda_{2} \rightarrow \mathbb{Z}_{N}$ is equal to the number of conjugacy classes of orbits of the action of $H$ on $X$.

Corollary 3.13 Let $X$ be either an annulus or a Möbius strip, with a single cone point of order $m \geq 2$. There exists an action of $G=\mathbb{Z}_{N}$ on $S$ with $S / G \approx X$ if and only if $N$ is even and $m$ divides $N$, and then the number of topological types of such action is

$$
\left\lceil\begin{array}{l}
\left.\frac{m \varphi(d)}{2 d}\right\rceil \begin{array}{l}
\text { if } d \neq 2 \text { or } 4 \nmid m, \\
\frac{m}{4}+1
\end{array} \text { if } d=2 \text { and } 4 \mid m,
\end{array}\right.
$$

where $d=(m, N / m)$. In particular, such action is unique up to topological conjugation if and only if $m \in\{2,3,4,6\}$ and if $m>2$ then $m^{2} \mid N$.

\subsection{Actions with a 2-punctured disc as the quotient orbifold}

We fix an NEC group $\Lambda$ with signature $\left(0 ;+;\left[m_{1}, m_{2}\right] ;\{()\}\right)$ and generators $x_{1}, x_{2}, c$, satisfying the following defining relations:

$$
x_{1}^{m_{1}}=x_{2}^{m_{2}}=c^{2}=1, \quad x_{1} x_{2} c=c x_{1} x_{2} .
$$

The following lemma is proved in [4, Proposition 4.10]

Lemma 3.14 If $m_{1} \neq m_{2}$ then $\operatorname{Out}(\Lambda)$ has order 2 and is generated by the class of automorphism $\alpha$, defined by

$$
\alpha:\left\{\begin{array}{l}
x_{1} \mapsto x_{1}^{-1} \\
x_{2} \mapsto x_{1} x_{2}^{-1} x_{1}^{-1} \\
c \mapsto c
\end{array}\right.
$$

The next one is proved in [2, Theorem 3.5 and Corollary 3.3] and it is also particular case of Lemma 5.16 in [10] for $r=2$.

Lemma 3.15 $(\sigma, N)$ is admissible if and only if $N=\operatorname{lcm}\left(m_{1}, m_{2}\right)$ and $N$ is even.

Proposition 3.16 Suppose that $(\sigma, N)$ is admissible, $m_{1} \neq m_{2}$ and $k=\left(m_{1}, m_{2}\right)$. There are exactly $\varphi(k)$ equivalence classes of $N S K$-maps $\Lambda \rightarrow \mathbb{Z}_{N}$.

Proof Every NSK-map $\theta: \Lambda \rightarrow \mathbb{Z}_{N}$ is equivalent (by multiplication by an element of $\mathbb{Z}_{N}^{*}$ ) to $\theta_{a}$ defined by $\theta_{a}(c)=\frac{N}{2}, \theta_{a}\left(x_{1}\right)=\frac{N}{m_{1}}$ and $\theta_{a}\left(x_{2}\right)=a \frac{N}{m_{2}}$ for some $a \in \mathbb{Z}_{m_{2}}^{*}$. We are going to show that $\theta_{a}$ is equivalent to $\theta_{a^{\prime}}$ if and only if $a \equiv a^{\prime}(k)$. 
Suppose that $\theta_{a}$ is equivalent to $\theta_{a^{\prime}}$. Then $\theta_{a}=b \theta_{a^{\prime}} \phi$ for some $b \in \mathbb{Z}_{N}^{*}$ and $\phi \in \operatorname{Aut}(\Lambda)$. By Lemma 3.14, for every $\phi \in \operatorname{Aut}(\Lambda)$ either $\phi\left(x_{i}\right)$ is conjugate to $x_{i}$ for $i=1,2$, or $\phi\left(x_{i}\right)$ is conjugate to $x_{i}^{-1}$ for $i=1,2$. It follows that $\theta_{a}\left(x_{i}\right)=b^{\prime} \theta_{a^{\prime}}\left(x_{i}\right)$ for $i=1,2$, where $b^{\prime}=b$ or $b^{\prime}=-b$. We have $b^{\prime} \equiv 1\left(m_{1}\right)$ and $b^{\prime} a^{\prime} \equiv a\left(m_{2}\right)$, hence $a^{\prime} \equiv a(k)$.

Conversely, suppose that $a^{\prime} \equiv a(k)$. By Lemma 2.5, there exists unique $b \in \mathbb{Z}_{N}^{*}$ such that $b \equiv 1\left(m_{1}\right)$ and $b \equiv\left(a^{\prime}\right)^{-1} a\left(m_{2}\right)$, where $\left(a^{\prime}\right)^{-1}$ is the inverse of $a^{\prime}$ in $\mathbb{Z}_{m_{2}}^{*}$. We have $\theta_{a}=b \theta_{a^{\prime}}$.

To finish the proof it suffices to note that for each $d \in \mathbb{Z}_{k}^{*}$ there exists $a \in \mathbb{Z}_{m_{2}}^{*}$ such that $d \equiv a(k)$. Hence, equivalence classes of NSK-maps $\Lambda \rightarrow \mathbb{Z}_{N}$ are in one to one correspondence with elements of $\mathbb{Z}_{k}^{*}$.

Remark 3.17 If $m_{1}=m_{2}=N$ then there exists $\phi \in \operatorname{Aut}(\Lambda)$ which swaps the conjugacy classes of $x_{1}$ and $x_{2}$. Consequently, $\theta_{a}$ and $\theta_{a^{\prime}}$ are equivalent if and only if either $a=a^{\prime}$ or $a a^{\prime}=1$. Hence, the number of equivalence classes of NSK-maps $\Lambda \rightarrow \mathbb{Z}_{N}$ is $\frac{\varphi(N)}{2}+z$, where $z$ is the number of square roots of 1 in $\mathbb{Z}_{N}^{*}$. Note that $z$ depends on the number of different prime divisors of $N$.

Corollary 3.18 There exists an action of $G=\mathbb{Z}_{N}$ on $S$ with $S / G$ being a disc with 2 cone points of distinct orders $m_{1}, m_{2}$ if and only if $N=\operatorname{lcm}\left(m_{1}, m_{2}\right)$ and $N$ is even, and then the number of topological types of such action is $\varphi(k)$, where $k=\left(m_{1}, m_{2}\right)$. In particular, such action is unique up to topological conjugation if and only if $k \leq 2$.

\subsection{Actions with a 2-punctured projective plane as the quotient orbifold}

In this subsection we fix $\Lambda$ with signature $\left(1 ;-;\left[m_{1}, m_{2}\right] ;\{-\}\right)$ and canonical generators $x_{1}, x_{2}, d$ of $\Lambda$, satisfying the following defining relations:

$$
x_{1}^{m_{1}}=x_{2}^{m_{2}}=1, \quad x_{1} x_{2} d^{2}=1 .
$$

Lemma 3.19 If $m_{1} \neq m_{2}$, then $\operatorname{Out}(\Lambda)$ is isomorphic to the Klein four-group and is generated by classes of automorphisms $\alpha, \beta$ defined by

$$
\alpha:\left\{\begin{array}{l}
x_{1} \mapsto x_{1} \\
x_{2} \mapsto\left(x_{2} d\right) x_{2}^{-1}\left(x_{2} d\right)^{-1} \\
d \mapsto x_{2} d
\end{array} \quad \beta:\left\{\begin{array}{l}
x_{1} \mapsto\left(x_{2} d\right)^{-1} x_{1}^{-1}\left(x_{2} d\right) \\
x_{2} \mapsto x_{2} \\
d \mapsto\left(d x_{2}\right)^{-1}
\end{array}\right.\right.
$$

Proof Recall from Sect. 2.2 that $\operatorname{Out}(\Lambda)$ is isomorphic to the pure mapping class group $\operatorname{PMod}\left(S,\left\{P_{1}, P_{2}\right\}\right)$, where $S=\mathcal{H} / \Lambda$ a projective plane with 2 distinguished points $P_{1}, P_{2} \in$ $S$ (Fig. 2). For $i=1,2$ let $V_{i}$ denote the isotopy class of the self-homeomorphism of $S$ obtained by sliding the puncture $P_{i}$ once along the loop $v_{i}$ on Fig. 2. By [14, Corollary 4.6] $\operatorname{PMod}\left(S,\left\{P_{1}, P_{2}\right\}\right)$ is generated by $V_{1}$ and $V_{2}$ and is isomorphic to $\mathbb{Z}_{2} \times \mathbb{Z}_{2}$. By computing

Fig. 2 Projective plane with 2 punctures, the loops of the puncture slides (left) and the generators of $\pi_{1}\left(S \backslash\left\{P_{1}, P_{2}\right\}\right)$ (right)
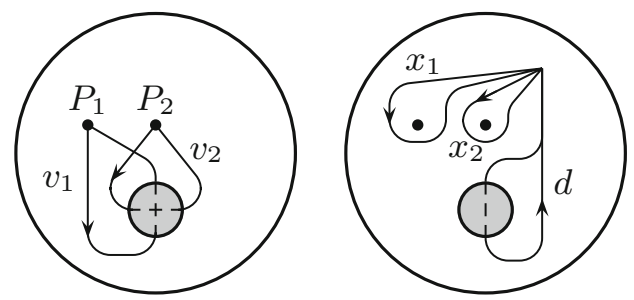
the automorphisms of $\pi_{1}\left(S \backslash\left\{P_{1}, P_{2}\right\}\right)$ induced by $V_{1}$ and $V_{2}$ we find that they represent the same elements of $\operatorname{Out}(\Lambda)$ as respectively $\beta$ and $\alpha$.

The next lemma is a special case of [10, Lemma 5.8] for $r=2$.

Lemma $3.20(\sigma, N)$ is admissible if and only if $N=1 \mathrm{~cm}\left(m_{1}, m_{2}\right)$ and $\frac{N}{m_{i}}$ are odd for $i=1,2$.

Proposition 3.21 Suppose that $(\sigma, N)$ is admissible, $N$ is odd, $m_{1} \neq m_{2}$ and $k=\left(m_{1}, m_{2}\right)$. There are exactly $\left[\frac{\varphi(k)}{2}\right]$ equivalence classes of NSK-maps $\Lambda \rightarrow \mathbb{Z}_{N}$.

Proof Every NSK-map $\theta: \Lambda \rightarrow \mathbb{Z}_{N}$ is equivalent (by multiplication by an element of $\mathbb{Z}_{N}^{*}$ ) to $\theta_{a}$ such that $\theta_{a}\left(x_{1}\right)=\frac{N}{m_{1}}$ and $\theta_{a}\left(x_{2}\right)=a \frac{N}{m_{2}}$ for some $a \in \mathbb{Z}_{m_{2}}^{*}$. Since $N$ is odd, $\theta_{a}(d)$ is determined by the relation $2 \theta_{a}(d)=-\left(\theta_{a}\left(x_{1}\right)+\theta_{a}\left(x_{2}\right)\right)$. Similarly as in the proof of Proposition 3.16 it can be shown that $\theta_{a}$ is equivalent to $\theta_{a^{\prime}}$ if and only if $a \equiv \pm a^{\prime}$ (k) (the only difference is that now $\Lambda$ admits an automorphism, e.g. $\alpha$ from Lemma 3.19, such that $\alpha\left(x_{1}\right)$ and $\alpha\left(x_{2}\right)$ are conjugate respectively to $x_{1}$ and $\left.x_{2}^{-1}\right)$. If $k>1$, then it is impossible that $a \equiv-a(k)$ for $a \in \mathbb{Z}_{m_{2}}^{*}$, because $k$ is odd. Hence, there are $\frac{\varphi(k)}{2}$ equivalence classes of NSK-maps if $k>1$, and one class if $k=1$.

Proposition 3.22 Suppose that $(\sigma, N)$ is admissible, $N$ is even, $m_{1} \neq m_{2}$ and $k=\left(m_{1}, m_{2}\right)$. There are exactly $\varphi(k)$ equivalence classes of NSK-maps $\Lambda \rightarrow \mathbb{Z}_{N}$.

Proof Let $\theta: \Lambda \rightarrow \mathbb{Z}_{N}$ be a NSK-map. After multiplication by an element of $\mathbb{Z}_{N}^{*}$ we may assume that $\theta\left(x_{1}\right)=\frac{N}{m_{1}}$ and $\theta\left(x_{2}\right)=a \frac{N}{m_{2}}$ for some $a \in \mathbb{Z}_{m_{2}}^{*}$. We have $2 \theta(d)+\theta\left(x_{1}\right)+$ $\theta\left(x_{2}\right)=0$, and since $N$ is even, $\theta(d)$ is determined by $\theta\left(x_{1}\right)$ and $\theta\left(x_{2}\right)$ only modulo $\frac{N}{2}$. Suppose that $\theta^{\prime}: \Lambda \rightarrow \mathbb{Z}_{N}$ is another NSK-map, such that $\theta^{\prime}\left(x_{1}\right)=\frac{N}{m_{1}}$ and $\theta^{\prime}\left(x_{2}\right)=a^{\prime} \frac{N}{m_{2}}$ for some $a^{\prime} \in \mathbb{Z}_{m_{2}}^{*}$. We claim that $\theta$ and $\theta^{\prime}$ are equivalent if and only if either

1. $a \equiv a^{\prime}(k)$ and $\theta^{\prime}(d)=b \theta(d)$, where $b$ is the unique element of $\mathbb{Z}_{N}^{*}$ satisfying $b \equiv 1\left(m_{1}\right)$ and $b a \equiv a^{\prime}\left(m_{2}\right)$, or

2. $a \equiv-a^{\prime}(k)$ and $\theta^{\prime}(d)=b\left(\theta(d)+\theta\left(x_{1}\right)\right)$, where $b$ is the unique element of $\mathbb{Z}_{N}^{*}$ satisfying $b \equiv-1\left(m_{1}\right)$ and $b a \equiv a^{\prime}\left(m_{2}\right)$.

To prove the claim we note $\theta$ and $\theta^{\prime}$ are equivalent if and only if $\theta^{\prime}=b \theta \phi$ for some $b \in \mathbb{Z}_{N}^{*}$ and $\phi \in \operatorname{Aut}(\Lambda)$. By Lemma 3.19 we may suppose that $\phi \in\{1, \alpha, \beta, \alpha \beta\}$. If $\phi=1$ or $\phi=\alpha \beta$, then after replacing $b$ by $-b$ in the latter case, we have $\theta^{\prime}\left(x_{i}\right)=b \theta\left(x_{i}\right)$ for $i=1,2$ and $\theta^{\prime}(d)=b \theta(d)$. Thus $b$ satisfies $b \equiv 1\left(m_{1}\right)$ and $b a \equiv a^{\prime}\left(m_{2}\right)$. By Lemma 2.5 , such (unique) $b$ exists if and only if $a^{\prime} a^{-1} \equiv 1(k)$ which is equivalent to $a \equiv a^{\prime}(k)$. Similarly, if $\phi=\beta$ or $\phi=\alpha$, then after replacing $b$ by $-b$ in the latter case, we have $\theta^{\prime}\left(x_{1}\right)=-b \theta\left(x_{1}\right), \theta^{\prime}\left(x_{2}\right)=b \theta\left(x_{2}\right)$ and $\theta^{\prime}(d)=b\left(\theta(d)+\theta\left(x_{1}\right)\right)$. Such (unique) $b$ exists if and only if $a \equiv-a^{\prime}(k)$. This completes the proof of the claim.

Suppose $k>2$. It follows from the previous paragraph that there is a surjection $\rho$ from the set of equivalence classes of NSK-maps onto $\mathbb{Z}_{k}^{*} /\{-1,1\}$, defined by $\rho([\theta])=\left[[a]_{k}\right]$, where $\theta$ is as above, $[\theta]$ is its equivalence class. We note that $\rho$ is 2 -to- 1 . Indeed, take $\theta$ and $\theta^{\prime}$ as above and suppose that they are not equivalent, but $\rho[\theta]=\rho\left[\theta^{\prime}\right]$. Then either

(1') $a \equiv a^{\prime}(k)$ and $\theta^{\prime}(d)=b \theta(d)+\frac{N}{2}$, where $b$ is as in (1) above, or

(2') $a \equiv-a^{\prime}(k)$ and $\theta^{\prime}(d)=b\left(\theta(d)+\theta\left(x_{1}\right)\right)+\frac{N}{2}$, where $b$ is as in (2) above. 
In case $\left(1^{\prime}\right), \theta^{\prime}$ is equivalent (by multiplication by $b^{-1}$ ) to $\theta_{1}$ defined by $\theta_{1}\left(x_{1}\right)=\frac{N}{m_{1}}$, $\theta_{1}\left(x_{2}\right)=a \frac{N}{m_{2}}, \theta_{1}(d)=\theta(d)+\frac{N}{2}$. In case $\left(2^{\prime}\right) \theta^{\prime}$ is equivalent (by multiplication by $-b^{-1}$ ) to $\theta_{2}$ defined by $\theta_{2}\left(x_{1}\right)=\frac{N}{m_{1}}, \theta_{2}\left(x_{2}\right)=-a \frac{N}{m_{2}}, \theta_{2}(d)=-\theta(d)-\frac{N}{m_{1}}+\frac{N}{2}$. Observe that $\theta_{1}$ and $\theta_{2}$ are equivalent [by (2) with $b=-1$ ], hence they represent the unique class $\left[\theta_{1}\right]$, such that $\left[\theta_{1}\right] \neq[\theta]$ and $\rho\left[\theta_{1}\right]=\rho[\theta]$. It follows that the number of equivalence classes of NSK-maps $\Lambda \rightarrow \mathbb{Z}_{N}$ is $2\left|\mathbb{Z}_{k}^{*} /\{-1,1\}\right|=\varphi(k)$.

Suppose $k=2$. By (1) every NSK map is equivalent to $\theta: \Lambda \rightarrow \mathbb{Z}_{N}$ such that $\theta\left(x_{1}\right)=\frac{N}{m_{1}}$ and $\theta\left(x_{2}\right)=\frac{N}{m_{2}}$. Fix such $\theta$ and define $\theta^{\prime}$ by $\theta^{\prime}\left(x_{i}\right)=\theta\left(x_{i}\right)$ for $i=1,2$ and $\theta^{\prime}(d)=$ $\theta(d)+\frac{N}{2}$. We have to show that $\theta$ and $\theta^{\prime}$ are equivalent. Let $b$ be the unique element of $\mathbb{Z}_{N}^{*}$ such that $b \equiv-1\left(m_{1}\right)$ and $b \equiv 1\left(m_{2}\right)$. By (2) it suffices to show that $\theta^{\prime}(d)=b\left(\theta(d)+\theta\left(x_{1}\right)\right)$. We have

$$
2 b \theta(d)=-b\left(\theta\left(x_{1}\right)+\theta\left(x_{2}\right)\right)=\theta\left(x_{1}\right)-\theta\left(x_{2}\right)=2\left(\theta\left(x_{1}\right)+\theta(d)\right)
$$

Either $b \theta(d)=\theta(d)+\theta\left(x_{1}\right)$ or $b \theta(d)=\theta(d)+\theta\left(x_{1}\right)+\frac{N}{2}$. The former equality is not possible, because $\theta\left(x_{1}\right)=\frac{N}{m_{1}}$ is odd (by Lemma 3.20) and $\theta(d)(b-1)$ is even. Hence

$$
b\left(\theta(d)+\theta\left(x_{1}\right)\right)=b \theta(d)-\theta\left(x_{1}\right)=\theta(d)+\frac{N}{2}=\theta^{\prime}(d)
$$

It follows that all NSK-maps $\Lambda \rightarrow \mathbb{Z}_{N}$ are equivalent.

Corollary 3.23 There exists an action of $G=\mathbb{Z}_{N}$ on $S$ with $S / G$ being a projective plane with 2 cone points of distinct orders $m_{1}, m_{2}$ if and only if $m_{1}, m_{2}$ and $N$ satisfy the conditions from Lemma 3.20, and then the number of topological types of such action is

$$
\begin{array}{ll}
\left\lceil\frac{\varphi(k)}{2}\right\rceil & \text { if } 2 \nmid N, \\
\varphi(k) & \text { if } 2 \mid N,
\end{array}
$$

where $k=\left(m_{1}, m_{2}\right)$. In particular, such action is unique up to topological conjugation if and only if $k \leq 3$.

\subsection{Actions with 3-punctured disc or projective plane as the quotient orbifold}

Here we assume that $p \leq q$ and $\frac{1}{p}+\frac{1}{q}>\frac{1}{2}$, which means that either $p=2$ or $(p, q) \in$ $\{(3,3),(3,4),(3,5)\}$. We fix NEC groups $\Lambda_{1}$ and $\Lambda_{2}$ with signatures $(0 ;+;[2, p, q] ;\{()\})$ and $(1 ;-;[2, p, q] ;\{-\})$, denoted respectively by $\sigma_{1}$ and $\sigma_{2}$. We also fix canonical generators $x_{1}, x_{2}, x_{3}, c$ of $\Lambda_{1}$, satisfying the following defining relations:

$$
x_{1}^{2}=x_{2}^{p}=x_{3}^{q}=c^{2}=1, \quad x_{1} x_{2} x_{3} c=c x_{1} x_{2} x_{3},
$$

and canonical generators $x_{1}, x_{2}, x_{3}, d$, of $\Lambda_{2}$, satisfying the following defining relations:

$$
x_{1}^{2}=x_{2}^{p}=x_{3}^{q}=1, \quad x_{1} x_{2} x_{3} d^{2}=1 .
$$

The next lemma follows immediately form Lemma 2.3 .

Lemma 3.24 $\left(\sigma_{1}, N\right)$ is admissible if and only if $N=\operatorname{lcm}(2, p, q)$.

Proposition 3.25 Suppose that $\left(\sigma_{1}, N\right)$ is admissible. If $(p, q) \neq(3,3)$ then $\left(\sigma_{1}, N\right)$ is rigid, whereas for $(p, q)=(3,3)$ there are 2 equivalence classes of NSK-maps $\Lambda_{1} \rightarrow \mathbb{Z}_{6}$. 
Proof Suppose that $\theta: \Lambda_{1} \rightarrow \mathbb{Z}_{N}$ is an NSK-map. We have $\theta\left(x_{1}\right)=\theta(c)=\frac{N}{2}, \theta\left(x_{2}\right)=k \frac{N}{p}$ for some $k \in \mathbb{Z}_{p}^{*}$ and $\theta\left(x_{3}\right)=l \frac{N}{q}$ for some $l \in \mathbb{Z}_{q}^{*}$.

If $p=2$ then $\theta\left(x_{2}\right)=\frac{N}{2}$, and by post-composing $\theta$ with an automorphism of $\mathbb{Z}_{N}$ we may assume $\theta\left(x_{3}\right)=\frac{N}{q}$. If $(p, q) \in\{(3,4),(3,5)\}$, then by using Chinese Remainder Theorem, we can find $c \in \mathbb{Z}_{N}^{*}$ such that $c \theta\left(x_{2}\right)=\frac{N}{p}$ and $c \theta\left(x_{3}\right)=\frac{N}{q}$. Finally if $(p, q)=(3,3)$ and $N=6$, then by post-composing $\theta$ with an automorphism of $\mathbb{Z}_{6}$ we may assume $\theta\left(x_{2}\right)=2$. There are two possibilities for $\theta\left(x_{3}\right): 2$ and 4, leading to non-equivalent NSK-maps (for one of them we have $\theta\left(x_{2}\right)=\theta\left(x_{3}\right)$, whereas for the other one $\left.\theta\left(x_{2}\right) \neq \theta\left(x_{3}\right)\right)$.

Corollary 3.26 Let $\frac{1}{p}+\frac{1}{q}>\frac{1}{2}$. There exists an action of $G=\mathbb{Z}_{N}$ on $S$ with $S / G$ being a disc with 3 cone points of orders $2, p, q$ if and only if $N=\operatorname{lcm}(2, p, q)$, and such action is unique up to topological conjugation if $(p, q) \neq(3,3)$, whereas for $(p, q)=(3,3)$ there are two topological types of such action.

Lemma $3.27\left(\sigma_{2}, N\right)$ is admissible if and only if $p=2, q$ is odd and $N=2 q$.

Proof Suppose that $\theta: \Lambda_{2} \rightarrow \mathbb{Z}_{N}$ is an NSK-map. Since $\Lambda^{+}$is generated by conjugates of $x_{i}$, condition (3) of Lemma 2.3 implies $N=\operatorname{lcm}(2, p, q)$. As in the proof of Proposition 3.25, we may assume that $\theta\left(x_{1}\right)=\frac{N}{2}, \theta\left(x_{2}\right)=\frac{N}{p}, \theta\left(x_{3}\right)=\frac{N}{q}\left[\right.$ or $\theta\left(x_{3}\right)=4$ if $\left.(p, q)=(3,3)\right]$. Set $z=\theta(d)$ and note that it has to satisfy the equation

$$
\theta\left(x_{1}\right)+\theta\left(x_{2}\right)+\theta\left(x_{3}\right)+2 z=0
$$

It is easy to check that such $z$ exists if and only if $p=2$ and $q$ is odd.

Proposition 3.28 If $\left(\sigma_{2}, N\right)$ is admissible then it is rigid.

Proof By the proof of Lemma 3.27, any NSK-map $\Lambda_{2} \rightarrow \mathbb{Z}_{N}$ is equivalent to one of $\theta_{i}$ defined for $i=1,2$ by $\theta_{i}\left(x_{1}\right)=\theta_{i}\left(x_{2}\right)=q, \theta_{i}\left(x_{3}\right)=2$ and $\theta_{1}(d)=-1, \theta_{2}(d)=q-1$. These are equivalent, as $\theta_{1}=\theta_{2} \circ \alpha$, where $\alpha$ is the following automorphism of $\Lambda_{2}$.

$$
\alpha:\left\{\begin{array}{l}
x_{1} \mapsto\left(x_{2} x_{3} d\right)^{-1} d^{-1}\left(x_{2} x_{3} d\right) \\
x_{2} \mapsto x_{2} \\
x_{3} \mapsto x_{3} \\
d \mapsto\left(d x_{2} x_{3}\right)^{-1}
\end{array}\right.
$$

Corollary 3.29 Let $\frac{1}{p}+\frac{1}{q}>\frac{1}{2}$. There exists an action of $G=\mathbb{Z}_{N}$ on $S$ with $S / G$ being a projective plane with 3 cone points of orders $2, p, q$ if and only if $p, q$ and $N$ satisfy the conditions from Lemma 3.27, and such action is unique up to topological conjugation.

\section{Self-homeomorphisms with large periods}

Let $C(g, N)$ denote the number of topological types of action of $\mathbb{Z}_{N}$ on a closed non-orientable surface of genus $g \geq 3$. It is proved in [2] that $C(g, N)=0$ for $N>2 g$, and if $g$ is even then $C(g, N)=0$ for $N>2(g-1)$. In this section we compute $C(g, N)$, for all $g \geq 3$ and $N>\max \left\{g, \frac{3}{2}(g-2)\right\}$.

For an admissible pair $(\sigma, N)$, let $c(\sigma, N)$ denote the number of equivalence classes of NSK-maps $\theta: \Lambda \rightarrow \mathbb{Z}_{N}$, where $\Lambda$ is an NEC-group with $\sigma(\Lambda)=\sigma$ (see Sect. 2 for 
definitions). Then $C(g, N)$ is the sum of all $c(\sigma, N)$ such that $(\sigma, N)$ is an admissible pair satisfying $N \mu(\sigma)=g-2$.

We begin by determining the possible signatures $\sigma$ of the form (2.2) satisfying $0<$ $\mu(\sigma)<\frac{2}{3}$. By a straightforward calculation we obtain the following list.

$$
\begin{aligned}
\sigma_{0} & =(0 ;+;[2,2,2] ;\{()\}), & & \sigma_{1}=(1 ;-;[2,2,2] ;\{-\}), \\
\sigma_{2} & =(0 ;+;[2, m] ;\{()\}), m>2, & & \sigma_{3}=(1 ;-;[2, m] ;\{-\}), m>2, \\
\sigma_{4} & =(0 ;+;[3, m] ;\{()\}), m>2, & & \sigma_{5}=(1 ;-;[3, m] ;\{-\}), m>2, \\
\sigma_{6} & =(0 ;+;[4, m] ;\{()\}), 11 \geq m \geq 4, & \sigma_{7} & =(1 ;-;[4, m] ;\{-\}), 11 \geq m \geq 4, \\
\sigma_{8} & =(0 ;+;[5, m] ;\{()\}), 7 \geq m \geq 5, & \sigma_{9} & =(1 ;-;[5, m] ;\{-\}), 7 \geq m \geq 5, \\
\sigma_{10} & =(0 ;+;[2] ;\{(),()\}), & & \sigma_{11}=(1 ;-;[2] ;\{()\}), \\
\sigma_{12} & =(2 ;-;[2] ;\{-\}) . & &
\end{aligned}
$$

In the cases where a signature $\sigma_{i}$ depends on the parameter $m$, we will also denote it as $\sigma_{i}(m)$. Now, for each of the above signatures we determine all admissible pairs $\left(\sigma_{i}, N\right)$, such that $N>g$, where $g=N \mu\left(\sigma_{i}\right)+2$.

Theorem 4.1 Let $g \geq 4$ and suppose that $(\sigma, N)$ is an admissible pair, such that $N>$ $\max \left\{g, \frac{3}{2}(g-2)\right\}$, where $g=N \mu(\sigma)+2$. Then $\sigma, N$ and $g$ are as in Table 1. Furthermore, $(\sigma, N)$ is rigid except for the following two cases

$$
\begin{aligned}
& c\left(\sigma_{4}(3(g-1) / 2), 3(g-1) / 2\right)=2 \text { for } g \equiv 1(4), \\
& c\left(\sigma_{6}(8), 8\right)=2 \text { for } g=7 .
\end{aligned}
$$

Table 1 All admissible pairs $(\sigma, N)$ such that $N>\max \{g, 3(g-2) / 2\}$

\begin{tabular}{llll}
\hline Signature $\sigma$ & Parameter $m$ & Order $N$ & Genus $g$ \\
\hline$\sigma_{2}$ & $g$ & $2 g$ & Odd \\
$\sigma_{2}$ & $2(g-1)$ & $2(g-1)$ & Arbitrary \\
$\sigma_{3}$ & $2(g-1)$ & $2(g-1)$ & Even \\
$\sigma_{4}$ & $3(g-1) / 2$ & $3(g-1) / 2$ & $g \equiv 1 \bmod 4$ \\
$\sigma_{4}$ & $(g+1) / 2$ & $3(g+1) / 2$ & $g \equiv 3 \operatorname{or} g \equiv 7 \bmod 12$ \\
$\sigma_{5}$ & $3(g-1) / 2$ & $3(g-1) / 2$ & $g \equiv 3 \bmod 4$ \\
$\sigma_{5}$ & $(g+1) / 2$ & $3(g+1) / 2$ & $g \equiv 1 \operatorname{or} g \equiv 9(12)$ \\
$\sigma_{6}$ & 6 & 12 & 9 \\
$\sigma_{6}$ & 7 & 28 & 19 \\
$\sigma_{6}$ & 8 & 8 & 7 \\
$\sigma_{6}$ & 9 & 36 & 25 \\
$\sigma_{6}$ & 10 & 20 & 15 \\
$\sigma_{6}$ & 11 & 44 & 31 \\
$\sigma_{8}$ & 6 & 30 & 21 \\
$\sigma_{9}$ & 7 & 35 & 25 \\
$\sigma_{10}$ & & $2(g-2)$ & $g>4$ \\
$\sigma_{11}$ & $2(g-2)$ & $g>4$ \\
$\sigma_{12}$ & & $2(g-2)$ & $g>4$ and $4 \mid g$ \\
\hline
\end{tabular}


Proof For $\sigma_{0}$ the only admissible pair is $\left(\sigma_{0}, 2\right)$ by Lemma 3.24, which does not satisfy $N>g$ as $N=2$ and $g=3$. For $\sigma_{1}$ there are no admissible pairs by Lemma 3.27.

By Lemma 3.15 a pair $\left(\sigma_{2}, N\right)$ is admissible if and only if $N=\operatorname{lcm}(2, m)$. Such pair is rigid for every $m$ by Proposition 3.16. We have $\mu\left(\sigma_{2}\right)=\frac{1}{2}-\frac{1}{m}$. If $m$ is odd then $N=2 m$ and $g=N \mu\left(\sigma_{2}\right)+2=m$. If $m$ is even then $N=m=2(g-1)$.

By Lemma 3.20 a pair $\left(\sigma_{3}, N\right)$ is admissible if and only if $N=m$ is even and $m / 2$ is odd. Every such pair is rigid by Proposition 3.22. We have $N=2(g-1)$ and $g$ is even.

By Lemma 3.15 a pair $\left(\sigma_{4}, N\right)$ is admissible if and only if $N=1 \mathrm{~cm}(3, m)$ and $m$ is even. We have $\mu\left(\sigma_{4}\right)=\frac{2}{3}-\frac{1}{m}$. If 3 divides $m$ then $N=m=\frac{3}{2}(g-1)$ and $g \equiv 1$ (4). In this case we have $c\left(\sigma_{4}, N\right)=\varphi(3)=2$ by Proposition 3.16. If $3 \nmid m$ then $N=3 m$ and $g=2 m-1$. We have $g \equiv 3$ or $g \equiv 7$ (12). In this case $\left(\sigma_{4}, N\right)$ is rigid by Proposition 3.16.

By Lemma 3.20 a pair $\left(\sigma_{5}, N\right)$ is admissible if and only if $N=\operatorname{lcm}(3, m)$ and $m$ is odd. For $m=3$ we have $N=g=3$, hence we assume $m>3$. Every such pair is rigid by Proposition 3.21. If 3 divides $m$ then $N=m=\frac{3}{2}(g-1)$ and $g \equiv 3$ (4). If $3 \nmid m$ then $N=3 m$ and $g=2 m-1$. We have $g \equiv 1$ or $g \equiv 9(12)$.

By Lemma 3.15 a pair $\left(\sigma_{6}, N\right)$ is admissible if and only if $N=\operatorname{lcm}(4, m)$. We have $\mu\left(\sigma_{2}\right)=\frac{3}{4}-\frac{1}{m}$. For $m=4$ we have $N=g=4$ which contradicts $N>g$. For $m \in\{7,9,11\}$ we have $N=4 m$ and $g=3 m-2$. For $m \in\{6,10\}$ we have $N=2 m$ and $g=\frac{3}{2} m$. For $m=8$ we have $N=8$ and $g=7$. By Proposition 3.16 we have $c\left(\sigma_{6}, 8\right)=\varphi(4)=2$ and $c\left(\sigma_{6}, m\right)=1$ for $m \neq 8$.

It follows from Lemma 3.20 that $\left(\sigma_{7}, N\right)$ is admissible if and only if $N=m, 4$ divides $m$ and $\frac{m}{4}$ is odd. This holds only for $m=4$, but then $N=g=4$ which contradicts $N>g$.

By Lemma 3.15 a pair $\left(\sigma_{8}, N\right)$ is admissible only for $m=6, N=30$ and $g=21$. By Proposition 3.16 this pair is rigid.

By Lemma 3.20 a pair $\left(\sigma_{9}, N\right)$ is admissible only for $m=N=5$ and $(m, N)=(7,35)$. In the former case we have $g=5$ which contradicts the assumption $N>g$. In the letter case we have $g=25$ and the pair is rigid by Proposition 3.22.

By Lemma 3.9 for $i \in\{10,11\}$ a pair $\left(\sigma_{i}, N\right)$ is admissible if and only if $N$ is even, and such pair is rigid by Proposition 3.12. We have $N=2(g-2)$. Note that $N>g$ only for $g>4$.

By Lemma 3.4 a pair $\left(\sigma_{12}, N\right)$ is admissible if and only if 4 divides $N$ and 8 does not divide $N$. Such pair is rigid by Proposition 3.5. We have $N=2(g-2)$ and 4 divides $g$.

Theorem 4.2 Suppose that $g \geq 11$ is odd and $g \notin\{15,19,21,25,31\}$. If $N>\frac{3}{2}(g-2)$ and $C(g, N)>0$ then

$$
N \in\left\{2 g, 2(g-1), 2(g-2), \frac{3}{2}(g+1), \frac{3}{2}(g-1)\right\} .
$$

Furthermore, $C(g, 2 g)=C(g, 2(g-1))=1, C(g, 2(g-2))=2$, and

$$
\begin{aligned}
& C\left(g, \frac{3}{2}(g+1)\right)= \begin{cases}1 & \text { for }[g]_{12} \in\{1,3,7,9\} \\
0 & \text { for }[g]_{12} \in\{5,11\}, g \neq 11\end{cases} \\
& C\left(g, \frac{3}{2}(g-1)\right)= \begin{cases}2 & \text { for } g \equiv 1(4), \\
1 & \text { for } g \equiv 3(4) .\end{cases}
\end{aligned}
$$

Proof The assumptions about $g$ guarantee that the numbers $2 g, 2(g-1), 2(g-2), \frac{3}{2}(g+1)$ and $\frac{3}{2}(g-1)$ are all different (the only exception is $g=11$, for which $\left.2(g-2)=\frac{3}{2}(g+1)\right)$, and also that there are no admissible pairs $\left(\sigma_{i}, N\right)$ for $i \in\{6,8,9\}$ with such $g$ in Table 1. 
Thus, the only possible values for $N$ are those given in the theorem. For each of these values we calculate $C(g, N)$ by adding up $c(\sigma, N)$ for all admissible pairs $(\sigma, N)$ from Table 1 .

$$
\begin{aligned}
& C(g, 2 g)=c\left(\sigma_{2}(g), 2 g\right)=1 \\
& C(g, 2(g-1))=c\left(\sigma_{2}(2(g-1)), 2(g-1)\right)=1 \\
& C(g, 2(g-2))=c\left(\sigma_{10}, 2(g-2)\right)+c\left(\sigma_{11}, 2(g-2)\right)=2
\end{aligned}
$$

For $N=3(g+1) / 2$ we have

$$
\begin{aligned}
& C(g, N)=c\left(\sigma_{4}(N / 3), N\right)=1 \text { for }[g]_{12} \in\{3,7\} \\
& C(g, N)=c\left(\sigma_{5}(N / 3), N\right)=1 \text { for }[g]_{12} \in\{1,9\}
\end{aligned}
$$

For $N=3(g-1) / 2$ we have

$$
\begin{aligned}
& C(g, N)=c\left(\sigma_{4}(N), N\right)=2 \text { for } g \equiv 1(4), \\
& C(g, N)=c\left(\sigma_{5}(N), N\right)=1 \text { for } g \equiv 3(4) .
\end{aligned}
$$

Theorem 4.3 Suppose that $g \geq 4$ is even. If $N>\frac{3}{2}(g-2), N>g$ and $C(g, N)>0$ then either $N=2(g-1)$ or $N=2(g-2)$. Furthermore, $C(g, 2(g-1))=2$ and for $g>4$

$$
C(g, 2(g-2))= \begin{cases}3 & \text { if } 4 \mid g, \\ 2 & \text { if } 4 \nmid g .\end{cases}
$$

Proof As in the proof of Theorem 4.2, we use Table 1 to compute $C(g, N)$. For $N=2(g-1)$ we have

$$
C(g, N)=c\left(\sigma_{2}(N), N\right)+c\left(\sigma_{3}(N), N\right)=2
$$

and for $N=2(g-2)$ and $g>4$

$$
\begin{array}{ll}
C(g, N)=c\left(\sigma_{10}, N\right)+c\left(\sigma_{11}, N\right)=2 & \text { if } 4 \nmid g, \\
C(g, N)=c\left(\sigma_{10}, N\right)+c\left(\sigma_{11}, N\right)+c\left(\sigma_{12}, N\right)=3 & \text { if } 4 \mid g .
\end{array}
$$

Theorem 4.4 The following is the complete list of values of $C(g, N)$ such that $N>g \geq 3$, $N>\frac{3}{2}(g-2)$ and $C(g, N)$ is not as in Theorem 4.2.

$$
\begin{aligned}
& C(5,6)=4, \quad C(7,12)=2, \quad C(7,8)=2, \quad C(9,12)=3, \quad C(15,20)=1, \\
& C(19,28)=1, C(21,30)=3, C(25,35)=1, C(25,36)=3, C(31,44)=1 \text {. }
\end{aligned}
$$

Proof We consider odd genera excluded by the assumption of Theorem 4.2. For $g=3$ we have only two admissible pairs with $N>g$, namely $\left(\sigma_{2}(3), 6\right)$ and $\left(\sigma_{2}(4), 4\right)$. Since each of them is rigid by Proposition 3.16, we have $C(3,4)=C(3,6)=1$, which agrees with Theorem 4.2. For $g=5$ we have $2(g-2)=\frac{3}{2}(g-1)=6$ and $C(5,6)=c\left(\sigma_{10}, 6\right)+c\left(\sigma_{11}, 6\right)+$ $c\left(\sigma_{4}(6), 6\right)=1+1+2=4$. For $g=7$ we have $2(g-1)=\frac{3}{2}(g+1)=12$ and $8<\frac{3}{2}(g-1)<$ $2(g-2) ; C(7,12)=c\left(\sigma_{2}(12), 12\right)+c\left(\sigma_{4}(4), 12\right)=2$ and $C(7,8)=c\left(\sigma_{6}(8), 8\right)=2$. For $g=9$ we have $12=\frac{3}{2}(g-1)$ and $C(9,12)=c\left(\sigma_{4}(12), 12\right)+c\left(\sigma_{6}(6), 12\right)=2+1=3$ For $g=15$ we have $20<\frac{3}{2}(g-1)$ and $C(15,20)=c\left(\sigma_{6}(10), 20\right)=1$. For $g=19$ we have $\frac{3}{2}(g-1)<28<\frac{3}{2}(g+1)$ and $C(19,28)=c\left(\sigma_{6}(7), 28\right)=1$. For $g=21$ we have $30=\frac{3}{2}(g-1)$ and $C(21,30)=c\left(\sigma_{4}(30), 30\right)+c\left(\sigma_{8}(6), 30\right)=2+1=3$. For $g=25$ we have $36=\frac{3}{2}(g-1)$ and $C(25,36)=c\left(\sigma_{4}(36), 36\right)+c\left(\sigma_{6}(9), 36\right)=3$ and $C(25,35)=$ $c\left(\sigma_{9}(7), 35\right)=1$. For $g=31$ we have $44<\frac{3}{2}(g-1)$ and $C(31,44)=c\left(\sigma_{6}(11), 44\right)=1$. 
Corollary 4.5 Suppose that $g \geq 5$ is odd and $g \not \equiv 5$ (12). Let $N$ be the maximum odd integer such that $C(g, N)>0$. Then

$$
N= \begin{cases}\frac{3}{2}(g+1) & \text { for } g \equiv 1(4), \\ \frac{3}{2}(g-1) & \text { for } g \equiv 3(4) .\end{cases}
$$

Furthermore, $C(g, N)=1$.

Acknowledgments The authors are grateful to referees for their helpful suggestions.

Open Access This article is distributed under the terms of the Creative Commons Attribution 4.0 International License (http://creativecommons.org/licenses/by/4.0/), which permits unrestricted use, distribution, and reproduction in any medium, provided you give appropriate credit to the original author(s) and the source, provide a link to the Creative Commons license, and indicate if changes were made.

\section{References}

1. Bagiński, C., Carvacho, M., Gromadzki, G., Hidalgo, R.: On periodic self-homeomorphisms of closed orientable surfaces determined by their orders. Collectanea Math. (2014, to appear)

2. Bujalance, E.: Cyclic groups of automorphisms of compact nonorientable Klein surfaces without boundary. Pac. J. Math. 109, 279-289 (1983)

3. Bujalance, E., Cirre, F.J., Conder, M.D.E.: Extensions of finite cyclic group actions on non-orientable surfaces. Trans. Am. Math. Soc. 365(8), 4209-4227 (2013)

4. Bujalance, E., Cirre, F.J., Conder, M.D.E., Szepietowski, B.: Finite group actions on bordered surfaces of small genus. J. Pure Appl. Algebra 214, 2165-2185 (2010)

5. Bujalance, E., Etayo J.J., Gamboa, J. M., Gromadzki, G.: Automorphisms groups of compact bordered Klein surfaces. A combinatorial approach. In: Lecture Notes in Mathematics, vol. 1439. Springer (1990)

6. Farb, B., Margalit, D.: A Primer on Mapping Class Groups. Princeton Mathematical Series 49, Princeton University Press, Princeton (2012)

7. Fujiwara, K.: On the outer automorphism group of a hyperbolic group. Isr. J. Math. 131, 277-284 (2002)

8. González-Acuña, F.J., Márquez-Bobadilla, J.M.: On the homeotopy group of the non orientable surface of genus three. Rev. Colombiana Mat. 40, 75-79 (2006)

9. Greenberg, L.: Maximal Fuchsian groups. Bull. Am. Math. Soc. 69, 569-573 (1963)

10. Gromadzki, G., Marzantowicz, W.: On conformal dynamics on orientable and nonorientable Riemann surfaces. Fund. Math 213, 169-190 (2011)

11. Harvey, W.J.: On branch loci in Teichmüller space. Trans. Am. Math. Soc. 153, 387-399 (1971)

12. Harvey, W.J., Maclachlan, C.: On mapping-class groups and Teichmüller spaces. Proc. Lond. Math. Soc. 30, 496-512 (1975)

13. Hirose, S.: On periodic maps over surfaces with large periods. Tohoku Math. J. 62(1), 45-53 (2010)

14. Korkmaz, M.: Mapping class groups of nonorientable surfaces. Geom. Dedicata 89, 109-103 (2002)

15. Nakagawa, K.: On the orders of automorphisms of a closed Riemann surface. Pac. J. Math. 115, 435-443 (1984)

16. Nielsen, J.: Die Struktur periodischer Transformationen von Flächen. Math. -fys. Medd. Danske Vid. Selsk 15(1) (1937); English translation in Jakob Nielsen collected works, 2, 65-102

17. Singerman, D.: Symmetries of Riemann surfaces with large automorphism group. Math. Ann. 210, 17-32 (1974)

18. Singerman, D.: Finitely maximal Fuchsian groups. J. Lond. Math. Soc. 6(2), 29-38 (1972)

19. Wang, S.: Maximum orders of periodic maps on closed surfaces. Topol. Appl. 41, 255-262 (1991) 